\title{
Impulsividad y búsqueda de sensaciones: factores asociados a síntomas de anorexia y bulimia nerviosas en estudiantes de secundaria
}

\section{Impulsiveness and sensation seeking: Factors associated with symptoms of anorexia and bulimia nervosa in high school students}

\author{
Serafina Castro-Zamudio ${ }^{1}$ y Josefina Castro-Barea ${ }^{2}$ \\ ${ }^{1}$ Facultad de Psicología, Universidad de Málaga, España \\ ${ }^{2}$ Distrito Sanitario Málaga-Guadalhorce, Servicio Andaluz de Salud, Málaga, España \\ Disponible online 31 de agosto de 2016
}

\begin{abstract}
El presente estudio examina la impulsividad y la búsqueda de sensaciones como posibles factores asociados a actitudes y comportamientos característicos de la anorexia y bulimia nerviosas en estudiantes de ambos sexos, entre 12 y 20 años de edad. El diseño se corresponde con un estudio observacional analítico de casos y controles, donde los casos corresponden al grupo de sujetos sintomáticos (han superado el punto de corte propuesto por los autores en los distintos instrumentos de evaluación) y los controles al grupo asintomático (no han superado el punto de corte). La muestra estaba constituida por 300 estudiantes de la provincia de Málaga (España), 136 varones (45.33\%) y 164 mujeres $(54.66 \%)$, que respondieron de manera voluntaria, anónima y bajo el consentimiento de los padres a los siguientes cuestionarios y escalas: Eating Disorder Inventory (EDI-II), Eating Attitudes Test (EAT-26), Bulimia Test Revised (BULIT-R), Barratt Impulsiveness Scale (BIS-11) y Sensation Seeking Scale (SSS-V). Los resultados constatan una correlación positiva entre la impulsividad y sintomatología asociada con la conducta alimentaria alterada, anorexia y bulimia nerviosas. En la variable búsqueda de sensaciones dicha correlación sólo es positiva cuando se estudia con sintomatología bulímica. En resumen, impulsividad y búsqueda de sensaciones parecen ser variables estrechamente relacionadas con los TCA y, por tanto, deberían ser incluidas en programas de estilos de vida saludables, ya que podrían contribuir a disminuir y/o prevenir el incremento de los TCA en población adolescente.
\end{abstract}

Palabras clave: Impulsividad; búsqueda de sensaciones; anorexia nerviosa, bulimia nerviosa; estudiantes.

\begin{abstract}
This study investigated the potential association of impulsiveness and sensation seeking and the attitudes and behaviour characteristic of anorexia and bulimia nervosa in male and female students (between 12 and 20 years). The study had an observational case-control design, in which the case group comprised symptomatic subjects who had scores above the cutoff point designated by the authors for several assessment instruments, and the control group, which comprised asymptomatic participants who had scores below the cutoff point. The study included 300 participants (136 men [45.33\%] and 164 women [(54.66\%]) from Malaga (Spain). All participants received parental authorization to take part in the study. The participants anonymously completed the following self-administered tests: Eating Disorder Inventory (EDI-II), Eating Attitudes Test (EAT-26), Bulimia Test Revised (BULIT-R), Barratt Impulsiveness Scale (BIS-11), and (SSS-V). The results suggest an association between impulsiveness and symptomatology associated with eating disorders, anorexia, and bulimia nervosa. In contrast, sensation seeking was only associated with bulimic symptoms. In summary, the variables impulsivity and sensation seeking appear to be closely associated with eating disorders. Thus, these aspects should be addressed in healthy lifestyle programs, because their inclusion may help to reduce or prevent the increase in eating disorders in the teenage population.
\end{abstract}

Key words: Impulsiveness; sensation seeking; anorexia nervosa; bulimia nervosa; students.

Correspondencia: Serafina Castro-Zamudio. Universidad de Málaga. Facultad de Psicología. Departamento de Personalidad, Evaluación y Tratamiento Psicológico. Campus de Teatinos s/n, 29071. Málaga. España. E-mail: scastro@uma.es. E-mail de la co-autora Josefina Castro-Barea: jcastrobarea@yahoo.es. 
En los últimos años se han producido cambios muy rápidos en los hábitos alimentarios de los españoles, observándose la misma evolución en el resto de países industrializados, con un incremento de las conductas alimentarias alteradas en los jóvenes, principalmente anorexia nerviosa (AN) y bulimia nerviosa (BN), así como un aumento significativo de comportamientos, actitudes y creencias relacionadas con estas alteraciones (PérezGaspar et al. 2000; Rivas, Bersabé y Castro, 2001; Echeburúa y Marañón, 2001; Ruiz-Lázaro, 2002; Toro, 2000).

Estos resultados vienen a corroborar los presentados en la Guía de Práctica Clínica sobre Trastornos de la Conducta Alimentaria del Sistema Nacional de Salud (2009), donde se realiza una revisión de los estudios presentados en España y se concluye que la población de mayor riesgo comprende a mujeres en la franja de edad de 12 a 21 años, y se obtiene una prevalencia del $0.14 \%$ al $0.9 \%$ para la $\mathrm{AN}$, del $0.41 \%$ al $2.9 \%$ para la $\mathrm{BN}$ y del $2.76 \%$ al $5.3 \%$ en el caso de los trastornos de la conducta alimentaria no especificados (TCANE). En total, se presentan unas cifras de prevalencia de los trastornos de la conducta alimentaria (TCA) del $4.1 \%$ al $6.41 \%$. En el caso de varones adolescentes, aunque existen menos estudios, la prevalencia sería del $0 \%$ para la $\mathrm{AN}$, del $0 \%$ al $0.36 \%$ para la BN y del $0.18 \%$ al $0.77 \%$ para los TCANE, con una prevalencia total de 0.27 a 0.90 .

El presente trabajo centra su interés en el estudio de dos factores como son la impulsividad y la búsqueda de sensaciones que, de evidenciar su papel en grupos sintomáticos afines a los trastornos de anorexia y bulimia nerviosas, deberían ser incluidos dentro de un programa que aborde aspectos tanto físicos como mentales, sociales y ambientales, promotores en definitiva de estilos de vida saludables que puedan contribuir a disminuir o prevenir el incremento de los TCA entre la población adolescente.

Siguiendo el concepto desarrollado por Dickman (1993), se entiende la impulsividad como un rasgo de personalidad multidimensional y complejo que se manifiesta en la conducta por una tendencia exagerada a realizar actos no planificados y, con frecuencia, socialmente inadecuados.

Peñas y Waller (2001) llevaron a cabo un estudio, con muestra no clínica, cuyo objetivo era comprobar si actitudes y comportamientos característicos de la $\mathrm{BN}$ mostraban puntos en común con un patrón de conducta impulsiva. Las participantes fueron 53 mujeres sin antecedentes de tratamiento psicológico (población no clínica) y los instrumentos de evaluación, The Bulimic Investigatory Test (BITE) y una escala para medir la conducta impulsiva. Los resultados mostraron que actitudes y comportamientos bulímicos en esta población se asociaban de forma generalizada con un patrón de conducta impulsiva.

Rosval et al. (2006) llevaron a cabo un estudio con una muestra de 84 mujeres diagnosticadas de BN, 37 de AN (19 de tipo restrictivo y 18 de tipo purgativo y con episodios de atracones) y 61 participantes sin trastorno, pertenecientes al grupo control. Solo las mujeres diagnosticadas de $\mathrm{BN}$ y $\mathrm{AN}$ de tipo purgativo mostraron puntuaciones altas en impulsividad motora, mientras que el grupo de mujeres diagnosticadas de bulimia nerviosa fue el único grupo que mostró tendencias hacia un comportamiento imprudente. Estos resultados sugieren que una conducta caracterizada por los atracones (BN y AN) correlaciona positivamente con problemas para el control de la impulsividad, mientras que una actitud de asumir riesgos puede ser una característica exclusiva de personas diagnosticadas de BN.

Guerrieri, Nederkoorn y Jansen (2007) investigaron el grado en que los rasgos de personalidad impulsiva, una dieta variada y la interacción entre ambas influían en la ingesta de alimentos. También estudiaron si la presencia de altos niveles de impulsividad se relacionaba positivamente con más pensamientos y conductas características de los TCA. Los resultados apuntaron en la dirección esperada, aquellas personas que presentaron un alto nivel de impulsividad ingerían más alimentos que aquellas con niveles más bajos, además de presentar más pensamientos y conductas relacionadas con los TCA.

Boisseau, Thompson-Brenner, Eddy y Satir (2009) y Moreno Domínguez, Ortega-Roldán y Rodríguez-Ruiz (2009) han llegado a las mismas conclusiones, la $\mathrm{BN}$ de tipo purgativo o la presencia de conducta purgativa en los TCA correlaciona de manera positiva con el rasgo de impulsividad. Además, Favaro et al. (2005) encontraron que los sujetos con AN de tipo restrictivo mostraban conductas impulsivas aunque a unos niveles más bajos que los grupos con atracones. De igual forma, este último grupo presentaba más impulsividad motora, sensación de excitación, menos autodisciplina y menos capacidad de reflexión en comparación con el grupo restrictivo (Claes, Vandereycken y Vertommen, 2005; Díaz-Marsá et al. 2008).

En otro estudio, Pascual-Jimeno, Apodaca-Urquijo, Etxebarria-Bilbao y Cruz-Sáez (2012), encontraron que el grupo de $\mathrm{BN}$ inespecífica (cumplen la mayoría de los criterios de acuerdo con el sistema de clasificación del DSM-IV-TR para una bulimia nerviosa) obtuvo puntuaciones más altas en impulsividad que el grupo de AN inespecífica (cumplen la mayoría de los criterios del DSM-IV-TR para una anorexia nerviosa). De igual modo, el grupo de BN no purgativa puntuaba más alto en impulsividad que el grupo de AN inespecífica. Por último, el grupo de BN purgativa también puntuó más alto en impulsividad cuando se comparó con el grupo de AN inespecífica. Por tanto, estos resultados corroboran que las mujeres con BN muestran mayor impulsividad que las mujeres con $\mathrm{AN}$.

Von Ranson, Wallace, Holub y Hodgins (2013), encontraron que algunas medidas de la impulsividad como los comportamientos imprudentes o la falta de planificación se asociaron significativamente con los juegos de azar y los TCA. Así, la impulsividad motora se asoció más específicamente con los juegos de azar y la impulsividad atencional se asoció más específicamente con los TCA. Los resultados encontrados en este estudio, sugieren que la comorbilidad entre estos dos trastornos puede estar influida, en parte, por la impulsividad elevada. Por lo tanto, una persona impulsiva con actitudes relacionadas con los TCA puede ser más propensa a desarrollar un TCA mientras que una persona menos impulsiva tendría menos probabilidades de desarrollar un TCA. 
Resultados similares se han encontrado en un estudio muy reciente de Farstad et al. (2015), donde concluyen que la impulsividad es un buen predictor de futuros atracones en la ingesta alimentaria así como de problemas con el juego. Por otro lado, también concluyen que la edad es un buen predictor de futuros atracones en las chicas más jóvenes frente a mujeres con mayor edad (el rango de edad oscilaba entre los 13 y 65 años y la muestra fue elegida de la población general).

La relación de la impulsividad con $\mathrm{BN}$ y trastornos afines es evidente. Se trata de un rasgo temperamental estrechamente relacionado con otros de naturaleza muy semejante, como es el caso del siguiente factor que contempla el presente estudio: la búsqueda de sensaciones, concepto ampliamente elaborado y difundido por Zuckerman (1979).

Entre las distintas investigaciones llevadas a cabo para estudiar el papel de este factor en AN y BN, se puede destacar el estudio planteado por Rossier, Bolognini, Plancherel y Halfon (2000) cuyo objetivo era conocer el rol de la búsqueda de sensaciones en los TCA. Los resultados mostraron que las participantes con $\mathrm{BN}$, cualquiera que fuera el subtipo de diagnóstico, obtuvieron mayores puntuaciones especialmente en la escala búsqueda de aventura y emoción. En contraposición, las participantes con AN de tipo restrictivo obtuvieron menor puntuación que el grupo control, especialmente en la escala de búsqueda de la experiencia.

Por otro lado, en una revisión sistemática llevada a cabo por Cassin y von Ranson (2005), encontraron diferencias consistentes entre la población anoréxica, caracterizada por un elevado control y persistencia y una baja búsqueda de la novedad, en comparación con la población bulímica que se caracterizaba por una alta impulsividad, búsqueda de sensaciones, búsqueda de la novedad y rasgos asociados al trastorno límite de la personalidad.

En el estudio realizado por Claes, Vandereycken y Vertommen (2005) los resultados mostraron también que los pacientes diagnosticados de $\mathrm{BN}$ mostraban mayor presencia de rasgo relativo a la urgencia y a la búsqueda de sensaciones, y menos a la perseverancia y premeditación en comparación con los pacientes diagnosticados de AN. Resultados similares se corroboran en un estudio posterior llevado a cabo por Claes et al. (2015) donde concluyeron que la urgencia ya sea positiva o negativa, la falta de premeditación y la falta de constancia eran significativamente mayores en los sujetos con conductas purgativas (AN de tipo purgativo, comedores compulsivos o $\mathrm{BN}$ ) frente a los sujetos del grupo control ("sanos") o con AN de tipo restrictivo.

En su mayoría, los estudios sobre TCA coinciden en obtener puntuaciones similares en determinados rasgos de personalidad. Sin embargo, al considerar distintos subtipos de TCA, encuentran una menor coincidencia en aspectos de temperamento y carácter: mientras que en $\mathrm{BN}$ se identifican elevadas puntuaciones en la escala de búsqueda de sensaciones (Álvarez-Moya et al. 2007; Fassino et al. 2002; Fernández-Aranda et al. 2006), en AN se obtiene elevada persistencia y bajas puntuaciones en la escala de búsqueda de sensaciones (Bloks, Hoek, Callewaert y van Furth 2004; Klump et al. 2000; Klump et al. 2004).

Basándonos en la evidencia arrojada por los distintos autores en los estudios anteriormente descritos, el objetivo de este trabajo consiste en hallar el grado de relación existente entre las variables objeto de estudio (impulsividad y búsqueda de sensaciones) y actitudes y comportamientos afines a la $\mathrm{AN}$ y $\mathrm{BN}$ en grupos sintomáticos (aquellos sujetos que obtienen puntuaciones por encima del punto de corte establecido en instrumentos como el EAT-26, EDI-II y BULIT-R) y grupo asintomático (puntuaciones por debajo del punto de corte en dichos instrumentos), esperando encontrar:

a) Correlación lineal positiva entre las puntuaciones obtenidas en el Test de Actitudes Alimentarias (EAT-26; Garner, Olmsted, Bohr y Garfinkel 1982) y las obtenidas en la Escala de Impulsividad de Barratt (BIS-11; Barrat 1994) o las obtenidas en la Escala de Búsqueda de Sensaciones ( SSS-V; Zuckerman, Eysenck y Eysenck 1978); esperando que el grupo sintomático, que resulte de superar el punto de corte en el EAT-26, muestre diferencias significativas en sintomatología relacionadas con la impulsividad y la búsqueda de sensaciones respecto del grupo asintomático (puntuación que no supera el punto de corte establecido por los autores).

b) Correlación lineal positiva entre las puntuaciones obtenidas en el Inventario de la Conducta Alimentaria (EDI-II; Garner 1991) y las obtenidas en el BIS-11 o en el SSS-V, esperando que el grupo sintomático, que resulte de superar el punto de corte en el EDI-II, muestre diferencias significativas en sintomatología relacionadas con la impulsividad y la búsqueda de sensaciones respecto del grupo asintomático (puntuación que no supera el punto de corte establecido por los autores).

c) Correlación lineal positiva entre las puntuaciones obtenidas en el Test de

Bulimia Revisado (BULIT-R; Thelen, Farmer, Wonderlich y Smith, 1991) y las obtenidas en el BIS-11 o en el SSS-V, esperando que el grupo sintomático, que resulte de superar el punto de corte en el BULIT-R, muestre diferencias significativas en sintomatología relacionadas con la impulsividad y la búsqueda de sensaciones respecto del grupo asintomático (puntuación que no supera el punto de corte establecido por los autores).

\section{Participantes}

\section{Método}

La muestra, no probabilística, intencional o de conveniencia y voluntaria, está compuesta por un total de 300 estudiantes de la provincia de Málaga (España), siendo el $45.33 \%$ chicos $(n=$ $136)$ y el $54.66 \%$ chicas $(n=164)$, con edades comprendidas entre 12 y 20 años de edad $(M=14.13$ y $D T=2.02)$, escolarizados en tres centros educativos entre los niveles de $1^{\circ}$ de ESO (Enseñanza Secundaria Obligatoria) y $2^{\circ}$ de Bachillerato y Ciclo Formativo. En todo momento se siguieron las normas éticas pertinentes. 


\section{Instrumentos de evaluación}

- Escala de Impulsividad de Barratt (Barratt Impulsiveness Scale, BIS-11; Barrat 1994; Oquendo et al. 2001). Es un cuestionario de 30 ítems con 4 opciones de respuesta sobre síntomas asociados a la impulsividad. Se divide en tres subescalas: impulsividad cognitiva, impulsividad motora e impulsividad no planeada. Desde el punto de vista clínico posee mayor relevancia el valor cuantitativo de la puntuación total que el de las subescalas. La validación española de Oquendo et al. (2001) ofrece una equivalencia lingüística, conceptual y de las escalas, correctas. La proporción de concordancia entre la versión inglesa y la castellana oscila entre .67 y .80 . No existe punto de corte propuesto. En varios estudios se sugiere utilizar como punto de corte la mediana de la distribución de la muestra estudiada.

- Escala de Búsqueda de Sensaciones (Sensation Seeking Scale, SSS-V; Pérez y Torrubia, 1986; Zuckerman et al. 1978). Es un cuestionario de 40 ítems de respuesta dicotómica. Se divide en cuatro subescalas: búsqueda de emociones, búsqueda de excitación, desinhibición y susceptibilidad hacia el aburrimiento. Puntuaciones elevadas en cada una de las subescalas, representan mayor presencia del rasgo. La fiabilidad test-retest en la validación española (Pérez y Torrubia, 1986) con un intervalo de 5 días es del .90 para la puntuación total y de entre .68 y .94 para las subescalas. Aunque no existen puntos de corte propuestos por los autores, facilitan las medias y desviaciones estándar obtenidas por la población normativa española: 21.3 (6.4) para los varones y 17.7 (5.3) para las mujeres.

- Test de Actitudes Alimentarias (Eating Attitudes Test, EAT-26; Garner, Olmstead, Bohr, y Garfinkel 1982; Castro, Toro y Salamero 1991). Es una escala de 26 ítems con 6 opciones de respuesta derivada de un análisis factorial del original EAT-40 (Garner y Garfinkel 1979). El hecho de crear el EAT-26 se debió al intento por solucionar el escaso poder predictivo del EAT-40 entre los subtipos restrictivo y bulímico de la AN. En general, existe un acuerdo en que este cuestionario es útil para identificar problemas alimentarios en poblaciones no clínicas además de ser sensible a los cambios que se producen como resultado del tratamiento (Chiodo 1985; Williamson 1990). Se compone de tres subescalas: hacer dieta, bulimia y preocupación por la comida y control oral. El punto de corte se establece en 20 (King 1989, 1991). La fiabilidad test-retest en un periodo de dos a tres semanas se sitúa en torno al $84 \%$. La sensibilidad y especificidad es del $95 \%$; el poder predictivo positivo es del $79 \%$ y del poder predictivo negativo del 94\% (Iñarritu, Cruz y Morán 2004).

- Inventario de Trastornos de la Conducta Alimentaria (Eating Disorder Inventory, EDI-II; Garner, 1991; Corral, González, Pereña, y Seisdedos 1998, adaptado por TEA). El EDI-II es un cuestionario de 91 ítems con 6 opciones de respuesta sobre síntomas normalmente asociados con la $\mathrm{AN}$ y BN. No pretende obtener un diagnóstico específico sino la observación y evaluación precisa de ciertos rasgos psicológicos o conjuntos de síntomas que, se supone, tienen relevancia para comprender y poder tratar los TCA. Está dividido en dos partes clínicamente relevantes, tres subescalas de actitudes hacia la alimentación y ocho subescalas disfuncionales personales. Puntuaciones altas para cada una de las subescalas se correspondería con un nivel mayor de psicopatología relacionada con la alimentación. La fiabilidad en grupos sanos oscila entre .29 y .88, para grupos de riesgo entre .31 y .88 y para grupos clínicos entre .69 y .90 . Las escalas del EDI-II se han diseñado para medir rasgos independientes aunque algunos autores sugieren un punto de corte sensible de 80 (García, Vázquez, López y Arcila 2003).

- Test de Bulimia Revisado (Bulimia Test Revised, BULITR; Berrios-Hernández et al. 2007). Se trata de una versión revisada del test original que desarrollaron Smith y Thelen en 1984 compuesto por 36 ítems con 5 opciones de respuesta, diseñado para evaluar los síntomas de la bulimia nerviosa basándose en los criterios diagnósticos del DSM-IV. En el análisis factorial se identificaron cinco dimensiones: atracones, control e imagen corporal, medidas radicales y ayuno, ejercicio físico, vómito y uso de laxantes y uso de diuréticos. La fiabilidad test-retest es del $95 \%$ con una alta consistencia interna (98\%) y una habilidad de falsos negativos del $80 \%$ y de falsos positivos del $40 \%$. La sensibilidad es del 91\% y la especificidad del $96 \%$ con un poder predictivo positivo del $81 \%$ y negativo del $98 \%$ (Welch, Thompson y Hall 1993). Se sugiere que los investigadores que utilicen este instrumento como prueba de tamizaje deberían utilizar un punto de corte de 85 para reducir el potencial de falsos negativos (Iñarritu et al., 2004).

\section{Procedimiento}

Una vez presentado y aprobado el estudio por parte de los distintos centros escolares, desde la propia dirección se envió una carta informando a los padres y tutores/as, y solicitando su consentimiento para la participación de aquellos alumnos/as menores de edad. Los cuestionarios fueron cumplimentados de manera voluntaria, de forma colectiva, anónima y en presencia de una psicóloga, con objeto de resolver las dudas que pudieran presentarse en el aula así como asegurar que los participantes respondieran a todos los ítems planteados en los instrumentos de evaluación. La duración de la prueba osciló entre 50 y 75 minutos.

El material se presentó en un único documento compuesto por los distintos cuestionarios, junto a un sobre en blanco, para que una vez cumplimentado el alumno/a pudiera introducirlo y cerrarlo, garantizando con ello la confidencialidad de las respuestas. No se eliminó a ningún participante del estudio ya que todos cumplimentaron de manera adecuada el 100\% de los ítems que forman cada uno de los cuestionarios.

\section{Análisis de datos}

El análisis estadístico se llevó a cabo mediante el paquete estadístico para Windows versión 15.0. En primer lugar, se rea- 
lizó un análisis exploratorio de los datos con el objetivo de detectar valores atípicos y extremos, detectar y etiquetar los valores perdidos y/o no aplicables así como describir la distribución de cada variable. Como todas las variables son cuantitativas, se calculó la media, desviación típica, mediana, mínimos, máximos y percentiles con el objetivo de corroborar y definir en la muestra estudiada los puntos de corte propuestos por los autores para cada instrumento de evaluación ya que el tipo de diseño se corresponde con un estudio observacional analítico de casos y controles, donde los casos, o grupo sintomático, se refieren a aquellos participantes que han igualado o superado los puntos de corte para cada instrumento de evaluación utilizado, y los controles, o grupo asintomático, a aquellos participantes que no lo han superado (véase instrumentos de evaluación).

Una vez descrita la muestra y corroborados los puntos de corte propuestos por los autores de los distintos instrumentos de evaluación utilizados, se continuó estudiando la normalidad de las variables cuantitativas aplicando el test de Kolmogorov-Smirnov y Shapiro Wilk. La única en la que se observa una distribución normal es la puntuación total arrojada por los participantes en la escala Búsqueda de Sensaciones (SSS-V) $(p=.07)$.

Para comenzar con el contraste de las distintas hipótesis planteadas en el estudio, se realizó un primer análisis de correlación bivariada aplicando el coeficiente de correlación de Spearman ya que todas las variables dependientes incluidas en este primer análisis de correlación son cuantitativas y no cumplen con el supuesto de normalidad.

Para completar el contraste de las distintas hipótesis formuladas se realizó una categorización de las variables cuantitativas continuas. Dicha categorización agrupa a aquellos participantes que han igualado o superado los puntos de corte propuestos para los distintos instrumentos en el grupo sintomático y aquellos que se encuentran por debajo de dicha puntuación en el grupo asintomático. Teniendo en cuenta los puntos de corte, se encontró que 21 participantes, es decir, el 7\% del total de la muestra, igualaron o superaron el punto de corte propuesto en el EAT-26; 35 participantes igualaron o superaron el punto de corte previsto en el EDI-II, es decir, el $11.7 \%$ y 12 son los participantes superaron el punto de corte previsto en el BULIT-R, es decir, el 4\% del total de la muestra. Para la Escala de Impulsividad 156 participantes igualaron o superaron el punto de corte propuesto en la muestra estudiada, es decir, el 52\% y, por último, para la Escala Búsqueda de Sensaciones han sido 162, es decir, el 54\% del total de la muestra (véase Figura 1).

Con objeto de evaluar si existen diferencias significativas entre aquellos sujetos que obtuvieron una puntuación igual o superior al punto de corte establecido para los distintos instrumentos de evaluación utilizados en TCA y aquellos que puntuaron por debajo, se aplicó la $U$ de Mann-Whitney.

Por último, para comprobar si existe esta misma diferencia entre grupos pero en la variable búsqueda de sensaciones (al distribuirse esta última según una norma) se aplicó una Prueba $T$ para muestras independientes.
Figura 1

Porcentaje de sujetos del grupo sintomático y asintomático.

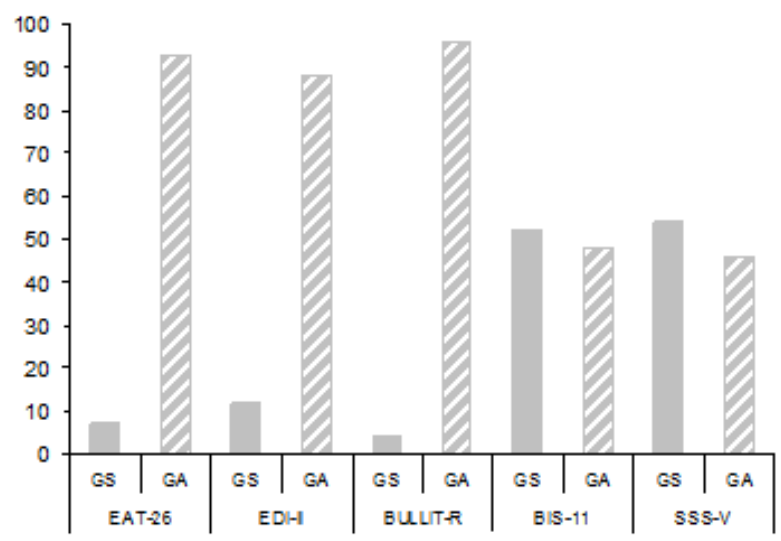

Nota. Test de Actitudes Alimentarias (EAT-26), Inventario de Trastornos de la Conducta Alimentaria (EDI-II), Test de Bulimia Revisado (BULITR), Escala de Impulsividad de Barrat (BIS-11), Escala de Búsqueda de Sensaciones (SSS-V), GS= Grupo sintomático, $\mathrm{GA}=$ Grupo asintomático.

\section{Resultados}

Los resultados obtenidos en el análisis exploratorio así como la definición de los puntos de corte pueden observarse en las Tablas 1 y 2 .

Tabla 1

Análisis descriptivo de la muestra

\begin{tabular}{lcccc}
\hline & Media $(D T)$ & Mediana & Mínimo & Máximo \\
\hline & $\begin{array}{c}\text { Total } \\
(N=300)\end{array}$ & $\begin{array}{c}\text { Total } \\
(N=300)\end{array}$ & $\begin{array}{c}\text { Total } \\
(N=300)\end{array}$ & $\begin{array}{c}\text { Total } \\
(N=300)\end{array}$ \\
\hline BIS-11 & $45.44(13.48)$ & $44^{*}$ & 17 & 85 \\
SSS-V & $16.67^{*}(5.97)$ & 17 & 2 & 37 \\
EAT-26 & $9.20(7.05)$ & 7 & 0 & 56 \\
EDI-II & $46.29(26.05)$ & 41 & 7 & 184 \\
BULIT-R & $55.032(14.34)$ & 52 & 36 & 108 \\
\hline
\end{tabular}

Nota. Escala de Impulsividad de Barratt (BIS-11), Escala de Búsqueda de Sensaciones (SSS-V), Test de Actitudes Alimentarias (EAT-26), Inventario de Trastornos de la Conducta Alimentaria (EDI-II), Test de Bulimia Revisado (BULIT-R).

* Equivalencia en la muestra estudiada de los puntos de corte propuestos por los autores del BIS-11 y el SSS-V.

Tabla 2

Percentiles de las puntuaciones obtenidas por la muestra en los instrumentos de evaluación de los TCA

\begin{tabular}{|llrrrlll}
\hline & 5 & 10 & 25 & 50 & 75 & 90 & 95 \\
\hline EAT-26 & 2 & 3 & 5 & 7 & 12 & 17 & $21^{*}$ \\
EDI-II & 15 & 18 & 27 & 41 & 59 & $81^{*}$ & 95 \\
BULIT-R & 38 & 40 & 43 & 52 & 63 & 74 & $82.95^{*}$ \\
\hline
\end{tabular}

Nota. Test de Actitudes Alimentarias (EAT-26), Inventario de Trastornos de la Conducta Alimentaria (EDI-II), Test de Bulimia Revisado (BULIT-R).

* Equivalencia en la muestra estudiada de los puntos de corte propuestos por los autores del EAT-26, EDI-II, BULIT-R.

Los resultados muestran una correlación lineal positiva entre las puntuaciones totales obtenidas por los participantes en el Inventario de Trastornos de la Conducta Alimentaria (EDI-II) y las obtenidas en el Test de Bulimia Revisado (BULIT-R) con las arrojadas por la Escala de Impulsividad de Barratt (BIS-11), así 
como las puntuaciones totales obtenidas en el Test de Bulimia Revisado (BULIT-R) con las arrojadas por la Escala de Búsqueda de Sensaciones (SSS-V), resultados que apoyan algunas de las hipótesis planteadas en este estudio (véase Tabla 3). La única variable que parece no correlacionar con la impulsividad y la búsqueda de sensaciones es la puntuación total obtenida por la muestra en el Test de Actitudes Alimentarias (EAT-26), test relacionado con la evaluación de aspectos más generales acerca del comportamiento alimentario alterado.

Tabla 3

Resultados de las correlaciones bivariadas

\begin{tabular}{|lccccc|}
\hline & EAT-26 & EDI-II & BULIT-R & BIS-11 & SSS-V \\
\hline EAT-26 & -- & -- & -- & 0.034 & 0.016 \\
EDI-II & -- & -- & -- & $0.205(* *)$ & 0.108 \\
BULIT-R & -- & -- & -- & $0.288(* *)$ & $0.313(* *)$ \\
BIS-11 & 0.034 & $0.205(* *)$ & $0.288(* *)$ & -- & -- \\
SSS-V & 0.016 & 0.108 & $0.313(* *)$ & -- & -- \\
\hline
\end{tabular}

Nota. Escala de Impulsividad de Barratt (BIS-11), Escala de Búsqueda de Sensaciones (SSS-V), Test de Actitudes Alimentarias (EAT-26), Inventario de Trastornos de la Conducta Alimentaria (EDI-II), Test de Bulimia Revisado (BULIT-R).

** La correlación es significativa al nivel .01 (bilateral).

La $U$ de Mann-Whitney aplicada con objeto de poder evaluar si existen diferencias significativas entre grupos en la variable impulsividad, apunta a que esta diferencia sólo es significativa para el EAT-26 $(p=.02 ; p<.05)$, resultado no observable en el anterior análisis de correlaciones.

La prueba $T$ para muestras independientes llevada a cabo para comprobar si existe esta diferencia entre grupos nos muestra que en la variable búsqueda de sensaciones, aunque la puntuación media es mayor para el grupo sintomático respecto del grupo asintomático, no podemos concluir que estas diferencias entre grupos sean significativas: en el EAT-26 $(M=17.76$; $D T=$ 4.89) vs. $(M=16.59 ; D T=6.04)$; en el EDI-II $(M=18.20 ; D T=$ 5.12) vs. $(M=16.47 ; D T=6.05)$; $\mathrm{y}$ finalmente en el BULIT-R $(M$ $=19.08 ; D T=7.16)$ vs. $(M=16.57 ; D T=5.91)$,

\section{Discusión}

El objetivo de este trabajo ha sido comprobar si la impulsividad y la búsqueda de sensaciones se pueden considerar factores asociados a actitudes y comportamientos característicos de los trastornos de la conducta alimentaria en una muestra de estudiantes de secundaria, ya que de ser así serían factores a tener en cuenta de cara a la prevención y/o intervención de un posible TCA. De hecho, los estudios descritos al principio de este trabajo concluyen que la sintomatología relacionada con los TCA, particularmente la ingesta compulsiva/purgativa, guarda mayor relación con la impulsividad y la búsqueda de sensaciones que con los comportamientos dirigidos a la restricción de la comida o al excesivo ejercicio físico. A nivel general, esta relación se establece para algunos de los síntomas relacionados con los TCA pero no para la totalidad de criterios diagnósticos y eso es algo que se constata en los estudios que hemos revisado y que se presentan a continuación.
Nuestros resultados están en la misma línea que los de Kinnetz (1998) cuyo estudio apuntaba que las mujeres con síntomas purgativos eran las que mayor puntuación presentaban en impulsividad. Por otro lado, también se alinean con los de Peñas y Waller (2001) quienes obtuvieron que actitudes y comportamientos bulímicos se asociaban de forma generalizada con un patrón de conducta impulsiva; en este estudio, se encontró una correlación lineal positiva y significativa entre las puntuaciones obtenidas por los participantes en la Escala de Impulsividad de Barratt y la sintomatología relacionada con la conducta alimentaria alterada, utilizando para ello el EDI-II y el BULIT-R. De igual forma se constató esta relación lineal positiva entre la impulsividad y las puntuaciones totales obtenidas por la población en los instrumentos de evaluación utilizados para evaluar sintomatología relacionada con los TCA en el estudio llevado a cabo por Guerrieri, Nederkoorn y Jansen (2007), quienes encontraron que aquellas personas que presentaban un alto nivel de impulsividad ingerían más alimentos que aquellas con niveles más bajos, además de presentar más pensamientos y conductas relacionadas con los TCA.

Otro grupo de autores cuyas conclusiones se asemejan a las encontradas en nuestro estudio son los de Boisseau et al. (2009), Moreno Domínguez et al. (2009) y Claes et al. (2015), dejando patente que la BN de tipo purgativo o la presencia de conducta purgativa en general en los TCA correlacionan de manera positiva con el rasgo de impulsividad frente a los sujetos con AN de tipo restrictivo o los sujetos de la población general o "sana".

Respecto a la variable búsqueda de sensaciones, los resultados encontrados en el presente estudio vienen a corroborar los ya encontrados por Rossier et al. (2000) o Claes et al. (2005), destacando la correlación lineal positiva entre la puntuación total arrojada por la muestra en la Escala de Búsqueda de Sensaciones (SSS-V) y las obtenidas por esta población en el BULIT-R. De igual forma, las investigaciones llevadas a cabo por Álvarez-Moya et al. (2007), Fassino et al. (2002) y Fernandez-Aranda et al. (2006) arrojaron puntuaciones elevadas en la escala de búsqueda de sensaciones en pacientes con BN, y las llevadas a cabo por Bloks et al. (2004) y Klump et al. (2004) obtuvieron elevada persistencia y bajas puntuaciones en la escala de búsqueda de sensaciones en pacientes con AN.

Aunque, como ya se ha señalado, lo descrito por la literatura científica parece ir en la línea de lo encontrado en este trabajo, se debe ser cauto a la hora de generalizar dichos resultados. Es importante considerar algunas de las limitaciones metodológicas incluidas en éste y otros estudios como, por ejemplo, aquellas relacionadas con la validez de las conductas autodeclaradas o la naturaleza no clínica de la población incluida. Dado el índice de prevalencia de estos trastornos y la difícil accesibilidad a la muestra, la mayoría de los estudios emplean cuestionarios de autoinforme para evaluar actitudes y comportamientos ante la alimentación, por lo que no puede establecerse a partir de ellos datos generalizables sino de población en riesgo de desarrollar el trastorno. 
Otra de las limitaciones a destacar es el tipo de diseño elegido para éste y otros estudios de fines parecidos cuando la muestra es no clínica, algo que no sólo compromete la validez externa, es decir, la posibilidad de generalizar los resultados obtenidos al resto de la población con similares características, sino que hace igualmente difícil poder establecer relaciones causales entre las variables objeto de estudio y los trastornos o enfermedades estudiadas. Esta es una de las causas por las que en este trabajo se tratan las distintas variables evaluadas como factores asociados a actitudes y comportamientos característicos de los trastornos de la conducta alimentaria y no como factores de riesgo para desarrollar anorexia, bulimia o cualquier otro TCA. Para poder hablar en términos de riesgo, no sólo se debe equiparar la muestra en términos de edad y género sino también utilizar diseños de pronóstico (cohorte) donde se pueda medir en el tiempo el efecto causal de la variable objeto de estudio en el desarrollo y/o mantenimiento de un determinado trastorno o etiológicos (casos y controles), donde los casos respondan a una verdadera muestra clínica. Por todo ello, es necesario realizar en nuestro entorno estudios con el suficiente rigor metodológico que nos permitan avanzar y realizar comparaciones con investigaciones realizadas previamente.

En definitiva, los resultados hallados en este estudio vienen a corroborar los encontrados en la literatura existente; no obstante, en el presente estudio no se ha utilizado una muestra clínica sino una muestra con adolescentes de la población general donde, a pesar de las limitaciones que presenta el estudio, se ha podido comprobar que aquellos sujetos con puntuaciones altas en impulsividad y búsqueda de sensaciones (por encima del punto de corte establecido por los autores) también obtienen puntuaciones por encima del punto de corte en instrumentos que evalúan TCA como son el EDI-II y BULIT-R; por tanto, los resultados de este trabajo son de interés para ayudar a entender la relación entre las variables objeto de este estudio. De igual modo, sería conveniente favorecer y/o promover, entre aquellos jóvenes que son más impulsivos o que presentan comportamientos imprudentes, estilos de vida saludables ya que podrían contribuir a disminuir o incluso prevenir el incremento de los TCA entre la población adolescente tan propensa a padecer este tipo de trastornos. En resumen, tal y como señalan algunos autores (Álvarez-Moya et al. 2007; Fassino et al. 2002; Fernández-Aranda et al. 2006; Laghi, Pompili, Baumgartner y Baiocco 2015; y Moreno Domínguez et al. 2009), la impulsividad así como la búsqueda de sensaciones pueden ayudar a detectar de forma precoz el riesgo a desarrollar un TCA y, por tanto, los programas de prevención deberían diseñarse para ayudar a los adolescentes a utilizar estrategias de adaptación que sean eficaces para manejar el sufrimiento y regular las emociones en lugar de utilizar comportamientos arriesgados para aliviar dicho dolor o sufrimiento.

Por último, queremos subrayar la importancia de realizar nuevos estudios que permitan determinar no sólo si variables como la impulsividad o la búsqueda de sensaciones pueden ser consideradas factores de riesgo en el desarrollo de un posible TCA sino también que permitan evaluar las diferencias que se dan entre estos dos factores y AN, BN o TCANE.

\section{Referencias}

1. Álvarez-Moya, E. M., Jimenez-Murcia, S., Granero, R., Vallejo, J., Krug, I., Bulik, C. M. y Fernández-Aranda, F. (2007). Comparison of personality risk factors in bulimia nervosa and pathological gambling. Comprehensive Psychiatry, 48, 452-457. http://dx.doi.org/10.1016/j.comppsych.2007.03.008

2. Barratt, E. S. (1994). Impulsiveness and aggression. En J. Monahan y H. J. Steadman (Eds.), Violence and mental disorder: Development in risk assessment (pp. 61-79). Chicago: The University of Chicago Press.

3. Berrios-Hernández M. N., Rodríguez-Ruiz S., Pérez M., Gleaves D. H., Maysonet M. y Cepeda-Benito A. (2007). Cross-cultural assessment of eating disorders: Psychometric properties of a Spanish version of the Bulimia TestRevised. European Eating Disorders Review, 15, 418-24. http://dx.doi.org/10.1002/erv.791

4. Bloks, H., Hoek, H. W., Callewaert, I. y van Furth, E. (2004). Stability of personality traits in patients who received intensive treatment for a severe eating disorder. The Journal of Nervous and Mental Disease, 192, 129-138. http://dx.doi.org/10.1097/01.nmd.0000110284.12816.fe

5. Boisseau, C. L., Thompson-Brenner, H., Eddy, K. T. y Satir, D. A. (2009). Impulsivity and Personality Variables in Adolescents with Eating Disorders. The Journal of Nervous and Mental Disease, 197, 251-259. http://dx.doi.org/10.1097/ NMD.0b013e31819d96c0

6. Cassin, S. E. y von Ranson, K. M. (2005). Personality and eating disorders: A decade in review. Clinical Psychology Review, 7, 895-916. http://dx.doi.org/10.1016/j. cpr.2005.04.012

7. Castro, J., Toro, J. y Salamero, M. (1991). The eating attitudes test: Validation of the Spanish version. Evaluación Psicológica, 7, 175-90.

8. Chiodo, J. (1985). The assessment of anorexia nervosa and bulimia nervosa. Progress in Behavior Modification, 19, 255-292. http://dx.doi.org/10.1016/B978-0-12-535619$\underline{0.50011-3}$

9. Claes, L., Islam, M. A., Fagundo, A. B., Jimenez-Murcia, S., Granero, R., Agüera, Z., Rosi, E., Menchón, J. M. y Fernández-Aranda, F. (2015). The Relationship between NonSuicidal Self-Injury and the UPPS-P Impulsivity Facets in Eating Disorders and Healthy Controls. PLoS ONE, 10(5), 1-11. http://dx.doi.org/10.1371/journal.pone.0126083

10. Claes, L., Vandereycken, W. y Vertommen, H. (2005). Impulsivity related traits in eating disorder patients. Personality and Individual Differences, 39, 739-749. http:// dx.doi.org/10.1016/j.paid.2005.02.022 
11. Corral, S., González, M., Pereña, J. y Seisdedos, N. (1998). Adaptación española del Inventario de trastornos de la conducta alimentaria. En D. M. Garner (Ed.), EDI-2: Inventario de Trastornos de la Conducta Alimentaria. Madrid: TEA.

12. Dickman, S. J. (1993). Impulsivity and information processing. En W. G. McCown, J.L. Johnson y M.B. Shure (Eds.), The impulsive client; theory, research, and treatment. Washington, DC: American Psychological Association. http://dx.doi.org/10.1037/10500-010

13. Díaz-Marsá, M., Carrasco, J. L., Basurte, E., Sáiz, J., López-Ibor, J. J. y Hollander, E. (2008). Enhanced cortisol supresión in Ealing disorders with impulsive personality features. Psychiatry Research, 158, 93-97. http://dx.doi. org/10.1016/j.psychres.2007.06.020

14. Echeburúa, E. y Marañón, I. (2001). Comorbilidad en las alteraciones de la conducta alimentaria con los trastornos de personalidad. Psicología Conductual, 9, 513-525.

15. Farstad, S. M., von Ranson, K. M., Hodgins, D. C., ElGuebaly, N., Casey, D. M. y Schopflocher, D. P. (2015). The influence of impulsiveness on binge eating and problem gambling: A prospective study of gender differences in Canadian adults. Psychology of Addictive Behaviors, 29, 805-812. http://dx.doi.org/10.1037/adb0000069

16. Fassino, S., Abbate-Daga, G., Amianto, F., Leombruni, P., Boggio, S. y Rovera, G. G. (2002). Temperament and character profile of eating disorders: a controlled study with the Temperament and Character Inventory. International Journal of Eating Disorders, 32, 412-425. http://dx.doi. org/10.1002/eat.10099

17. Favaro, A., Zanetti, T., Tenconi, E., Degortes, D., Ronzan, A., Veronese, A. y Santonastaso, P. (2005). The relationship between temperament and impulsive behaviors in eating disordered subjects. Eating Disorders, 13, 61-70. http:// dx.doi.org/10.1080/10640260590893647

18. Fernández-Aranda, F., Jiménez-Murcia, S., Álvarez, E., Granero, R., Vallejo, J. y Bulik, C. M. (2006). Impulse control disorders in eating disorders: Clinical and therapeutic implications. Comprehensive Psychiatry, 47, 482-488. http://dx.doi.org/10.1016/j.comppsych.2006.03.002

19. García, E., Vázquez, V., López, J. C. y Arcila, D. (2003). Validez interna y utilidad diagnóstica del Eating Disorders Inventory en mujeres mexicanas. Salud pública de México, 45, 206-210. http://dx.doi.org/10.1590/s003636342003000300010

20. Garner, D. M. (1991). Eating Disorder Inventory-2 Professional Manual. Odessa, Florida: Psychological Assessment Resources.

21. Garner, D. M. y Garfinkel, P. E. (1979). The eating attitudes test: An index of the symptoms of anorexia nervosa. Psychological Medicine, 9, 273-279. http://dx.doi.org/10.1017/ $\underline{\mathrm{S} 0033291700030762}$
22. Garner, D. M., Olmstead, M. P., Bohr, Y. y Garfinkel, P. E. (1982). The Eating attitudes test: Psychometric features and clinical correlates. Psychological Medicine, 12, 871-878. http://dx.doi.org/10.1017/S0033291700049163

23. Grupo de trabajo de la Guía de Práctica Clínica sobre Trastornos de la Conducta Alimentaria (2009). Guía de Práctica Clínica sobre Trastornos de la Conducta Alimentaria. Madrid: Plan de Calidad para el Sistema Nacional de Salud del Ministerio de Sanidad y Consumo. Agència d'Avaluació de Tecnologia i Recerca Mèdiques de Cataluña. Guías de Práctica Clínica en el SNS: AATRM Núm. 2006/05-01. Recuperado de http://www.guiasalud.es/GPC/ GPC 440 Tt_Conduc_Alim_compl_(4 jun).pdf

24. Guerrrieri, R., Nederkoorn, C. y Jansen, A. (2007). How impulsiveness and variety influence food intake in a sample of healthy women. Appetite, 48, 119-122. http://dx.doi. org/10.1016/j.appet.2006.06.004

25. Iñarritu, M. C., Cruz, V. y Morán, I. C. (2004). Instrumentos de evaluación para los trastornos de la conducta alimentaria. Revista de Salud Pública y Nutrición, 5, 1-7.

26. Kinnetz, P. L. (1998). Impulsivity in women with eating disorders. Disertación doctoral no publicada, Universidad de Cincinnati, Ohio, EE.UU.

27. King, M. B. (1989). Eating disorders in a general practice population. Prevalence, characteristics and follow-up at 12 to 18 months. Psychological Medicine, 14, 1-34. http:// dx.doi.org/10.1017/s0264180100000515

28. King, M. B. (1991). The natural history of eating pathology in attenders to primary medical care. International Journal of Eating Disorders, 10, 379-387.http://dx.doi. org/10.1002/1098-108X(199107) 10:4<379::AIDEAT2260100402>3.0.CO;2-I

29. Klump, K. L., Bulik, C. M., Pollice, C., Halmi, K. A., Fichter, M. M., Berrettini, W. H., Devlin, B., Strober, M., Kaplan, A., Woodside, D. B., Treasure, J., Shabbout, M., Lilenfeld, L. R. R., Plotnicov, K. H. y Kaye, W. H. (2000). Temperament and character in women with anorexia nervosa. The Journal of Nervous and Mental Disease, 188, 559-567. http://dx.doi.org/10.1097/00005053-200009000$\underline{00001}$

30. Klump, K.L., Strober, M., Johnson, C., Thornton, L., Bulik, C.M., Devlin, B., Fichter, M.M., Halmi, K.A., Kaplan, A.S., Woodside, D.B., Crow, S., Mitchell, J., Rotondo, A., Keel, P., Berrettini, W.H., Plotnicov, K., Pollice, C., Lilenfeld, L.R., Kaye, W.H. (2004). Personality characteristics of women before and after recovery from an eating disorder. Psychological Medicine, 34, 1407-1418. http://dx.doi. org/10.1017/S0033291704002442

31. Laghi, F., Pompili, S., Baumgartner, E. y Baiocco, R. (2015). The role of sensation seeking and motivations for eating in female and male adolescents who binge eat. 
Eating Behaviors, 17, 119-124. http://dx.doi.org/10.1016/j. eatbeh.2015.01.011

32. Moreno Domínguez, S., Ortega-Roldán, B. y RodríguezRuiz, S. (2009). Impulsividad en mujeres con bulimia nerviosa. eduPsykhé, 8, 63-77.

33. Oquendo, M. A., Baca-Garcia, E.,Graver, R., Morales, M., Montalban, V. y Mann, J. J. (2001). Spanish adaptation of the Barratt Impulsiveness Scale (BIS). European Journal of Psychiatry, 15, 147-155.

34. Pascual-Jimeno, A.; Apodaca-Urguijo, P., Etxebarria-Bilbao, I. y Cruz-Sáez, M. S. (2012). Diferencias en la frecuencia de experiencias emocionales positivas y negativas en mujeres con distintos tipos de trastornos de la conducta alimentaria. Anales de Psicología, 28, 743-752. http:// dx.doi.org/10.6018/analesps.28.3.156041

35. Peñas, E. y Waller, G. (2001). Bulimic psychopathology and impulsive behaviors among nonclinical women. International Journal of Eating Disorders, 29, 71-75. http:// dx.doi.org/10.1002/1098-108X(200101)29:1<71::AIDEAT11>3.0.CO;2-W

36. Pérez-Gaspar, M., Gual, P., de Irala-Estévez, J., MartínezGonzález, M. A., Lahortiga, F. y Cervera, S. (2000). Prevalencia de trastornos de la conducta alimentaria en las adolescentes navarras. Medicina Clínica, 114, 481-486. http:// dx.doi.org/10.1016/S0025-7753(00)71340-6

37. Pérez, J. y Torrubia, R. (1986). Fiabilidad y validez de la versión española de la escala de búsqueda de sensaciones (Forma V). Revista Latinoamericana de psicología, 18, 7-22.

38. Rivas, T., Bersabé, R. y Castro, S. (2001). Prevalencia de los trastornos de la conducta alimentaria en adolescentes de Málaga (España). Salud Mental, 24, 25-31.

39. Rossier, V., Bolognini, M., Plancherel. B. y Halfon O. (2000). Sensation seeking: A personality trait characteristic of adolescent girls and young women with eating disorders? European Eating Disorders Review, 8, 245-252. http://dx.doi. org/10.1002/(SICI)1099-0968(200005)8:3<245::AIDERV308>3.0.CO;2-D

40. Rosval, L., Steiger, H., Bruce, K., Israel, M., Richardson, J. y Aubut, M. (2006). Impulsivity in women with Ealing disorders: Problem of response inhibition, planning, or attention? International Journal of Eating Disorderes, 39, 590-593. http://dx.doi.org/10.1002/eat.20296
41. Ruiz-Lázaro, P. M. (2002). Epidemiología de los trastornos de la conducta alimentaria en España. Actas Españolas de Psiquiatría, 31, 85-94.

42. Smith, M. C. y Thelen, M. H. (1984). Development and validation of a test for bulimia. Journal Consulting Clinical Psychology, 21, 167-179.

43. Thelen, M. H., Farmer, J., Wonderlich, D. y Smith, M. (1991). A revision of the bulimia test: The BULIT-R. Journal consulting Clinical Psychology, 3, 119-124. http:// dx.doi.org/10.1037/1040-3590.3.1.119

44. Toro, J. (2000). La epidemiología de los trastornos de la conducta alimentaria. Medicina Clínica, 114, 543-544. http://dx.doi.org/10.1016/S0025-7753(00)71357-1

45. von Ranson K. M. , Wallace L. M. , Holub A. y Hodgins, D. C. (2013). Eating disorders, substance use disorders, and impulsiveness among disordered gamblers in a community sample. European Eating Disorders Review, 21, 148-154. http://dx.doi.org/10.1002/erv.2207

46. Welch, G., Thompson, L. y Hall, A. (1993). The BULIT$\mathrm{R}$ its realiability and clinical validity as a screening tool for DSM-III-R bulimia nervosa in a female tertiary education population. International Journal of Eating Disorder, 14, 95-105. http://dx.doi.org/10.1002/1098108X(199307)14:1<95::AID-EAT2260140113>3.0.CO;2Z

47. Williamson, D.A. (1990). Assesment of eating disorders: Obesity, anorexia and bulimia nervosa. New York: Pergamon Press.

48. Zuckerman, M. (1979). Sensation seeking: Beyond the optimal level of arousal. Hillsdale, NJ: Erlbaum.

49. Zuckerman, M., Eysenck, S. y Eysenck, H. (1978). Sensation seeking in England and America: Cross-cultural, age, and sex comparisons. Journal Consulting and Clinical Psychology, 46, 139-149. http://dx.doi.org/10.1037/0022006X.46.1.139

Fecha de recepción: 16 de septiembre, 2015

Fecha de recepción de la versión modificada: 1 de junio, 2016 Fecha de aceptación: 27 de junio, 2016 